\title{
COMPOSITION ALGEBRAS OF POLYNOMIALS
}

\author{
J. L. BRENNER
}

Dedicated to the memory of Ernst G. Straus

Briefly, a composition algebra $A$ involves two operations: addition and composition (substitution of polynomials). Let $C$ be an arbitrary commutative ring, and $C[x, y, \ldots]$ the ring of polynomials in the indeterminates $x, y, \ldots$ with coefficients from $C$. Addition of polynomials is commutative; composition is associative, and is distributive (on one side) over addition. (Notice that if the number of indeterminates is greater than 1, the operation of composition is not a binary operation.) We find the ideal structure of $A$ in some special cases. In particular, the ideals of $A$ are all principal (generated by a single element) if $C$ is a principal ideal ring (e.g. Z) and the number of variables is $1: A=\left(C[x],+,{ }^{\circ}\right)$, provided further that for all $c \in C, 2 \mid c+c^{2}$. [An example is the algebraic integers in $Q(\sqrt{-7})$.]

We start in a general context. An ideal $J$ in $A$ is the kernel of a homomorphism. Thus $J$ enjoys these three properties:

1.01. $J$ is a module over $C:$ If $c_{1}, c_{2} \in C, t_{1}, t_{2} \in J$, then $c_{1} t_{1}+c_{2} t_{2}$ $\in J$.

1.02. If $t \in J$ and $n_{1}, n_{2}, \ldots \in A$, then $t(x, y, \ldots) \circ\left[n_{1}, n_{2}, \ldots\right] \equiv$ $t\left(n_{1}, n_{2}, \ldots\right)$ lies in $J$.

1.03. If $t_{2}, t_{3}, \ldots \in J$ and if $n_{1}, n_{2}, \ldots \in A$, then $n_{1}(x, y, \ldots) \circ$ $\left[n_{2}+t_{2}, n_{3}+t_{3}, \ldots\right]-\left(n_{1}(x, y, \ldots) \circ\left[n_{2}, n_{3}, \ldots\right]\right)$ lies in $J$.

Since $n_{1}$ is a sum of monomials, it follows from 1.01 that 1.03 can be replaced by the simpler requirement

1.04 .

$$
\Pi_{i=2}^{k}\left(n_{i}+t_{l}\right)^{\alpha_{1}}-\Pi_{i=2}^{k} n_{i}^{\alpha_{2}} \quad \text { lies in } J
$$

1.05. Definition. An ideal $J=\langle a\rangle$ in $A$ is principal if $J$ is the smallest ideal containing $a . A$ is a principal ideal composition algebra (in short, $A$ is principal) if every ideal is principal. 
Even if $C$ is a principal ideal ring (say $C=\mathbf{Z}$ ) it can be seen that $A$ is not necessarily principal, so the property of being principal is not inherited. Recall the same situation in ordinary ring theory: $\mathbf{Z}[x, y]$ is not a principal ideal ring.

When the number of indeterminates is 1 , the situation is more tractable.

2. Ideals in the composition algebra $A=(\mathbf{Z}[x],+, \circ)$. In this section, all ideals in $A$ are described, in case the number of variables is 1 , and in case $C=\mathbf{Z}$. "Described" means that the additive basis for $J$ is given, $J$ being taken as a module over $C$. It turns out that $A$ is principal in this case. At bottom, the proof depends on a result in [1]. The present paper, by its dedication, recalls the contribution of E. G. Straus as referee of [1].

Note that if the number of variables is $1, A$ is a near ring. The characterization of an ideal $J$ in $A$ specializes as follows.

2.01. If $t_{1}, t_{2} \in J$ then $t_{1}+t_{2} \in J$.

2.02. If $t_{1} \in J$ and $n \in A$, then $t_{1} \circ n \in J$.

2.03. If $\alpha \geq 1, t \in J, n \in A$, then

$$
(n+t)^{\alpha}-n^{\alpha} \text { lies in } J .
$$

2.04. Lemma. If $t$ lies in the ideal $J, \alpha \geq 1$, then $t^{\alpha}$ lies in $J$.

Proof. Take $n=0$ in 2.03 .

2.05. Corollary $(n+t)^{\alpha}-n^{\alpha}-t^{\alpha}$ lies in $J$.

2.06. LEMMA. If $n_{1}$ is any polynomial, and if $t$ lies in the ideal $J$, then $n_{1}(t)-n_{1}(0)$ lies in $J$.

Proof. If $n_{1}(x)=\sum_{0}^{k} a_{\alpha} x^{\alpha}$, then $n_{1}(t)-n_{1}(0)=\sum_{1}^{k} a_{\alpha} t^{\alpha}$. Use 2.04 .

The next series of lemmas is directed to finding the smallest ideal $J(1)$ that contains 1 .

2.07. LemMA. $J(1)$ contains $2 x^{\nu}$ for $\nu=1,2, \ldots$

Proof. Use 2.05 with $n=x^{\nu}, t=1, \alpha=2:\left(x^{\nu}+1\right)^{2}-\left(x^{\nu}\right)^{2}-1=$ $2 x^{\nu}$ 
2.08. LEMMA. $J(1)$ contains $x^{2}+x$.

Proof. Use 2.05 with $\alpha=3, t=1, n=x$, together with 2.07.

2.09. LeMmA. Modulo $J(1), x^{\nu} \equiv x^{2 \nu} \equiv x^{4 v} \equiv \cdots \equiv x^{2^{s} \nu}, s=1,2, \ldots$; $\nu=1,2, \ldots$

Proof. $\left(x+x^{2}\right) \circ x^{\nu}=x^{\nu}+x^{2 \nu}$.

2.10. Corollary. $J(1)$ contains $x+x^{4}, x+x^{8}, x^{2}+x^{8}, x^{20}+x^{5}$, $x^{3}+x^{6}, x^{19}+x^{38}$.

2.11. LEMMA. $J(1)$ contains $x^{5}+x, x^{35}+x^{7}, x^{25}+x^{5}, x^{25}+x$.

Proof. $\left(x+x^{2}\right)^{3} \equiv x^{3}+x^{4}+x^{5}+x^{6} \equiv x+x^{5}$.

2.12. LEMMA. $J(1)$ contains $x+x^{17}$.

Proof. $\left(x+x^{4}\right)^{5}-\left(x+x^{4}\right) \circ x^{5} \equiv x^{8}+x^{17}$.

2.13. LeMMA. $J(1)$ contains $x+x^{19}$.

Proof. $\left(x^{4}+x^{17}\right)^{3}-\left(x^{4}+x^{17}\right) \circ x^{3} \equiv x^{25}+x^{38}$. Use 2.11.

2.14. LeMmA. $J(1)$ contains $x^{7}+x^{19}$.

Proof. $\left(x+x^{17}\right)^{3}-\left(x+x^{17}\right) \circ x^{3} \equiv x^{19}+x^{35}$.

2.15. LEMMA. $J(1)$ contains $x+x^{7}$.

Proof. Combine 2.13, 2.14.

2.16. ThEOREM. For $\nu=1,2, \ldots, J(1)$ contains $x^{3 \nu+1}+x, x^{3 \nu-1}+x$.

Proof by induction. For $\nu=1,2$ Theorem 2.16 is already proved. Suppose $\mu \geq 3$. Then

$$
\left(x^{4}+x^{3 \mu-4}\right)^{3}-\left(x^{4}+x^{3 \mu-4}\right) \circ x^{3} \equiv x^{3 \mu+4}+x^{6 \mu-4} .
$$

By induction hypothesis, $x+x^{3 \mu-2}$ lies in $J(1)$, so also does $\left(x+x^{3 \mu-2}\right) \circ x^{2}=x^{2}+x^{6 \mu-4}$. Hence $x^{3 \mu+4}+x^{2}$ lies in $J(1)$. Similarly, $\left(x^{2}+x^{3 \mu-2}\right)^{3}-\left(x^{2}+x^{3 \mu-2}\right) \circ x^{3} \equiv x^{3 \mu+2}+x^{6 \mu-2} \quad$ lies in $J(1)$; 
and

$$
x^{3 \mu+2}+x^{6 \mu-2}-\left(x+x^{3 \mu-1}\right) \circ x^{2} \equiv x^{3 \mu+2}+x^{2} .
$$

The inductive step from $x^{3 \mu \pm 1}+x$ to $x^{3 \mu+6 \pm 1}+x^{2}$ has been completed.

2.17. ThEOREM. $J(1)$ contains $x^{3 \nu}+x^{3}$ for $\nu=1,2 \ldots$

Proof. $x^{3}+x^{6}=\left(x+x^{2}\right) \circ x^{3} ; x^{3}+x^{9} \equiv\left(x+x^{4}\right)^{3}$ $-\left(x+x^{2}\right) \circ x^{6}$. If $3 \nu$ is a power of 3 , then $x^{3 \nu}+x^{9}=\left(x^{\nu}+x^{3}\right) \circ x^{3}$. This gives an inductive proof, since $x^{3 \nu}+x^{3} \equiv x^{3 \nu}+x^{9}+\left(x^{9}+x^{3}\right)$.

Suppose $3 \nu$ is not a power of 3 , say $3 \nu=\mu \rho$ with $\mu$ a power of 3 $(\mu \geq 3)$ and $\rho$ prime to $3, \rho>1$. Then $x^{\mu \rho}+x^{3} \equiv x^{\mu \rho}+x^{\mu}=\left(x^{\rho}+x\right) \circ$ $x^{\mu}$.

2.18. THEOREM. The module $J(1)$ with basis

$$
\left\langle 1,2 x^{\nu}, x^{3 \nu+1}+x, x^{3 \nu-1}+x, x^{3 \nu}+x^{3} \mid \nu=1,2, \ldots\right\rangle
$$

is an ideal in $(\mathbf{Z}[x],+, \circ)$.

Proof. Under the natural mapping $\mathbf{Z}[x] \rightarrow \mathbf{Z}_{2}[x]$, the module $J(1)$ is mapped into the ideal $J$ of [1]. Thus $J(1)$ is the full inverse image of an ideal, so $J(1)$ is an ideal. (A second proof is given in $\$ 4$.)

2.19. REMARK. 2.18 can be used to derive certain properties of binomial and multinomial coefficients.

2.20. REMARK. It turns out that $J(1)$ is multiplicatively closed.

2.21. THEOREM. Each of the modules

$$
\begin{aligned}
& V(1)=\left\langle 1,2 x^{\nu}, x^{\nu}+x \mid \nu=1,2, \ldots\right\rangle \\
& T(1)=\left\langle 1,2 x^{\nu}, x^{3 \nu+1}+x, x^{3 \nu-1}+x, x^{3 \nu} \mid \nu=1,2, \ldots\right\rangle
\end{aligned}
$$

is an ideal.

See [1].

The proofs of $2.22-2.25$ are left to the reader.

2.22. TheOREM. If $c$ is any integer, $c J(1), c T(1), c V(1)$ are ideals. $c J(1)=J(c)$ is the smallest ideal that contains $c$. If $c, d$ are constants, $c \mid d$, then $J(d) \subset J(c), d V(1)=V(d) \subset V(c), d T(1)=T(d) \subset T(c)$ (just as, in ring theory, $(d) \subset(c))$. 
2.23. THEOREM. $c \mathbf{Z}[x], T(c), V(c), J(c)$ are the only ideals in $\left(\mathbf{Z}[x],+,{ }^{\circ}\right)$, with inclusion relations given in 2.22 , and in the diagram

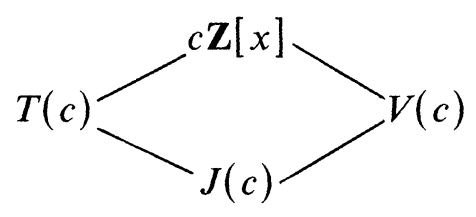

2.24. THEOREM. Every ideal $J$ in $A$ is closed under multiplication, that is, if $t_{1}, t_{2} \in J$ then $t_{1} t_{2} \in J$.

2.25. THEOREM. $\left(\mathbf{Z}[x],+,{ }^{\circ}\right)$ is a principal-ideal composition algebra, that is, a principal ideal near ring.

3. Ideals in the composition algebra $A_{m}=\left(\mathbf{Z}_{m}[x],+,{ }^{\circ}\right)$ when $m$ is odd. If $m$ is an odd prime, the ideals in $A_{m}$ were given in [2]. If $m$ is odd and composite, the results are similar but not identical. The proof of Lemma 3.01 appears in [2].

3.01. Lemma. Every (near-ring) ideal in $A_{m}$ is a ring ideal in the polynomial ring $\mathbf{Z}_{m}[x]$.

Proof. Let $J$ be an ideal in $A_{m}, f \in J$. It has to be proved that if $g \in A_{m}$, then $f g \in J$. First, $(f+g)^{2}-g^{2}-f^{2}=2 f g \in J$, because of $2.03,2.04$. Next, 2 is invertible, and by $2.06,\left(\frac{1}{2} x\right) \circ(2 f g)-\frac{1}{2} 0 \equiv f g$ lies in $J$.

If the ideal $J$ contains a nonzero constant $c, \S 2$ describes $J$. If the ideal $J$ contains a polynomial that is not 0 at every place in $\mathbf{Z}_{m}$, then $J$ contains a nonzero constant, by 2.02 . Hence the only interesting ideals in $A_{m}$ consist of polynomials that take only the value 0 ; because of 3.01 , each such ideal has a single "basis." Note that the (single polynomial) basis is the generator of a module with coefficients from $\mathbf{Z}_{m}[x]$ (not from $\mathbf{Z}_{m}$ as in §).

We first examine the case $m=p^{r}, p$ an odd prime, $r \geq 1$. If the ideal $J$ contains a (nonzero) constant $c$, then $J$ contains $c \mathbf{Z}_{m}[x]$. Otherwise, every polynomial in $J$ is 0 at every place in $\mathbf{Z}_{m}$. Every such polynomial is a multiple of a distinguished one, $f_{p, r}(x)$, of lowest degree:

$$
f_{p, r}(x)=x^{r}\left(x^{\phi\left(p^{r}\right)}-1\right) \text {. }
$$

This assertion follows from the fact that if two polynomials vanish at a place, so also does their gcd. We have to consider the ring ideals $\left(n_{1}(x) f_{p, r}(x)\right)$. 
3.02. LEMMA. If $n_{1}(x)$ is an arbitrary polynomial in $\mathbf{Z}_{m}[x]$, then the ring ideal $\left(n_{1}(x) f_{p, r}(x)\right)$ is an ideal in the composition algebra $A_{m}$.

Proof. The properties to check are 2.01, 2.02, 2.03. 2.01 is obviously satisfied. As for 2.02, note that

$$
\begin{aligned}
\left(n_{1}(x) f_{p, r}(x)\right) \circ n(x) & =n_{1}(n(x)) f_{p, r}(n(x)) \\
& =n_{1}(n(x)) n(x)^{r}\left(n(x)^{\phi\left(p^{r}\right)}-1\right) .
\end{aligned}
$$

It can be checked that the last factor is a multiple of $x^{\phi\left(p^{r}\right)}-1$, so that 2.02 is satisfied. This leaves 2.03. Here, the binomial theorem shows that

$$
\left(n(x)+n_{1}(x) f_{p, r}(x)\right)^{\alpha}-n(x)^{\alpha}
$$

is a multiple of $n_{1}(x) f_{p, r}(x)$, and Lemma 3.02 is verified.

If $m$ is odd but not a prime power, criteria 2.01-2.03 must be adjusted. 2.01 must be changed to read

3.021. If $c_{1}, c_{2} \in \mathbf{Z}_{m}$ and $t_{1}, t_{2} \in J$, then $c_{1} t_{1}+c_{2} t_{2} \in J$. The conditions 3.021, 2.02, 2.03 characterize ideals in $A_{m}$.

If $m$ is odd, there are ideals in $A_{m}$ that contain nonzero constants. Such an ideal can be a homomorphic image of $J(c)$, or can be the union of such images (since $\mathbf{Z}_{m}$ is not necessarily a principal ideal ring).

The interesting ideals in $A_{m}$ are a little more complicated to describe when $m$ is divisible by several primes. The difficulty lies just in finding the polynomials of lowest degree that are zero at every place in $\mathbf{Z}_{m}$. Suppose

$$
m=p_{1}^{\alpha(1)} \cdots p_{k}^{\alpha(k)} \text {. }
$$

Then a polynomial $f$ of lowest degree that is zero at every place in $\mathbf{Z}_{m}$ is

$$
f_{m}(x)=\operatorname{LCM}\left[f_{p_{1}, \alpha(1)}(x), \ldots, f_{p_{k}, \alpha(k)}(x)\right] .
$$

The rest of the theory is unaltered.

If $m$ is even, it is not true that every ideal in the composition algebra $A_{m}$ is a ring ideal in $\mathbf{Z}_{m}[x]$. See [2], where the case $m=2$ (among others) is fully discussed.

3.03. Problem. Describe the ideals in $A_{m}$ if $m$ is even.

4. Ideals in the composition algebra $A=\left(C[x],+,{ }^{\circ}\right)$ if $C$ is a principal ideal ring. Even if $C$ is a principal ideal ring, the ideals in $A=\left(C[x],+,{ }^{\circ}\right)$ can be hard to describe. The task is much simplified in the presence of the additional condition 4.01 . 
4.01. Condition. If $c \in C$, then $c+c^{2}$ is a multiple of 2 .

Note that this condition is trivially satisfied if 2 is invertible; but there are many rings in which 4.01 is satisfied, but 2 is not invertible. For instance, let $C$ be the ring of algebraic integers in $Q(\sqrt{m}), m$ squarefree. If $m$ is odd, the ring $C$ is the set

$$
\left\{\frac{1}{2}(a+b \sqrt{m}) \mid a, b \text { of the same parity }\right\} .
$$

The condition $2 \mid c+c^{2}$ requires that $m$ satisfy the further condition $m \equiv 1$ $\bmod 4$.

In the lemmas and theorems of this section, 4.01 is assumed to hold. We try to characterize the smallest ideal $J(c)$ that contains $c$.

4.02. Lemma. If an ideal $J$ in $A$ contains $f$, and if $c \in C$, then $J$ contains $c f$.

Proof. $(c x) \circ f-(c x) \circ 0=c f$. See 3.01 .

4.03. Lemma. If the ideal $J(c)$ in $A$ contains the constant $c$, then $J(c)$ contains $2 c x^{\nu}, \nu=1,2, \ldots$

Proof. $\left(x^{\nu}+c\right)^{2}-x^{2 \nu}-c^{2}=2 c x^{\nu}$.

4.04. LeMMA. $J(c)$ contains $c x^{2}+c^{2} x$.

Proof. $(x+c)^{3}-x^{3}-c^{3} \equiv c x^{2}+c^{2} x$.

4.05. COROLlaRy. $J(c)$ contains $c\left(x^{2}+x\right)$.

Proof. $c\left(x^{2}+x\right) \equiv c x^{2}+c^{2} x+\left(c+c^{2}\right) x$.

4.06. REMARK. $J(c)$ contains $\left(c^{\mu}+c^{\nu}\right) x^{\sigma}, \mu \geq \nu \geq 1, \sigma \geq 1$.

4.07. THEOREM. $J(c)$ contains $c J(1)$.

The proof is parallel to the proof of the corresponding result in $\$ 2$.

It is not obvious that $J(1)$ is an ideal in the present context; this has to be proved. A direct argument follows, based on several lemmas.

4.08. Lemma. J(1) is multiplicatively closed. Moreover,

$$
x^{3} \circ\left\langle x^{3 v+1}+x, x^{3 \nu-1}+x, x^{3 v}+x^{3}\right\rangle \subset J(1) .
$$


4.09. Remark. None of $V(1), T(1), J(1)$ is an ordinary ring ideal.

4.10. LemMa. If $n(x)$ is any polynomial, then each of $x^{3 v+1}+x$, $x^{3 v-1}+x, x^{3 v}+x^{3}$ admits multiplication by $(n(x))^{3}$.

Proof. Take $n(x)=\sum_{0}^{k} a_{i} x^{i}$. Then $(n(x))^{2} \equiv \sum_{0}^{k} a_{i}^{2} x^{2 i} \bmod 2$, that is, the two sides of the congruence differ by twice some polynomial. Hence

$$
(n(x))^{3} \equiv\left(\sum_{0}^{k} a_{i} x^{2 i}\right)\left(\sum_{0}^{k} a_{J} x^{j}\right) \bmod 2,
$$

since by hypothesis, $2 \mid a_{i}+a_{l}^{2}$. The product can be computed. Some of the terms are $a_{t}^{2} x^{3 i}(0 \leq i \leq k)$; see second assertion of Lemma 4.08. The remaining terms in the product occur in pairs: $a_{i} a_{j}\left(x^{2 i+j}+x^{2 j+i}\right)$. The two exponents are either both prime to 3 or both divisible by 3 , since their sum is $(2 i+j)+(2 j+i)=3(i+j)$. The lemma is proved.

4.11. LeMMA. If $n$ is an arbitrary polynomial, then $\left(x^{2}+x\right) \circ n(x)=$ $(n(x))^{2}+n(x)$ lies in J(1) and its constant term is divisible by 2 .

Proof. Take $n(x)=\sum_{0}^{k} a_{\imath} x^{\imath}$. Then

$$
(n(x))^{2}+n(x) \equiv \sum_{0}^{k}\left(a_{l} x^{2 i}+a_{i}^{2} x^{l}\right) \equiv \sum_{0}^{k} a_{i}\left(x^{2 i}+x^{i}\right) .
$$

4.12. Lemma. $\left(x^{4}+x^{2}\right) \circ n(x)$ lies in $J(1)$.

Proof. $\left(x^{4}+x^{2}\right) \circ n(x)=\left(x^{2}+x\right) \circ x^{2} \circ n(x)$.

4.13. Lemma. $\left(x^{5}+x^{4}\right) \circ n(x)$ lies in $J(1)$.

Proof. $(n(x))^{5}+(n(x))^{4}=(n(x))^{3}\left[(n(x))^{2}+n(x)\right]$. According to 4.11, the [ ] lies in $J(1)$ and has zero constant term. Apply 4.10.

4.14. Corollary. $\left(x^{5}+x\right) \circ n(x)$ lies in $J(1)$.

Proof. $x^{5}+x=\left(x^{5}+x^{4}\right)+\left(x^{4}+x^{2}\right)+\left(x^{2}+x\right)$. Apply 4.11, 4.12, 4.13 .

The last four lemmas can be generalized.

4.15. Lemma. if $m$ is composite and not divisible by 3 , then $\left(x^{m}+x\right) \circ n(x)$ lies in $J(1)$. 
Proof. Set $m=u v, 1<u \leq v<m$. Then

$$
\begin{aligned}
\left(x^{m}+x\right) \circ n(x) & \equiv\left(x^{u v}+x^{u}+x^{u}+x\right) \circ n(x) \\
& =\left(x^{u v}+x^{u}\right) \circ n(x)+\left(x^{u}+x\right) \circ n(x) \\
& =\left(x^{v}+x\right) \circ x^{u} \circ n(x)+\left(x^{u}+x\right) \circ n(x) .
\end{aligned}
$$

The terms lie separately in $J(1)$ by an obvious induction hypothesis, based on 4.16 .

4.16. LEMMA. $\left(x^{3 \nu+1}+x\right) \circ n(x),\left(x^{3 \nu+2}+x\right) \circ n(x)$ lie in $J(1)$.

Proof.

$$
\begin{gathered}
\left(x^{3 \nu+1}+x^{4}\right) \circ n(x)=(n(x))^{3}\left((n(x))^{3 v-2}+n(x)\right) \\
\left(x^{3 v+2}+x^{4}\right) \circ n(x)=(n(x))^{3}\left(n(x)^{3 v-1}+n(x)\right) .
\end{gathered}
$$

Apply a suitable induction hypothesis, together with 4.10 .

4.17. LEMMA. $\left(x^{6}+x^{3}\right) \circ n(x)$ lies in $J(1)$.

Proof. $\left(x^{6}+x^{3}\right) \circ n(x)=\left(x^{2}+x\right) \circ x^{3} \circ n(x) .$. Apply 4.11.

4.18. LEMMA. $\left(x^{9}+x^{6}\right) \circ n(x)$ lies in $J(1)$.

Proof. $\left(x^{9}+x^{6}\right) \circ n(x)=(n(x))^{3}\left[n(x)^{6}+n(x)^{3}\right]$. Apply 4.17, 4.10.

4.19. LeMma. If $\nu>3,\left(x^{3 \nu}+x^{6}\right) \circ n(x)$ lies in $J(1)$.

Proof.

$$
\left(x^{3 \nu}+x^{6}\right) \circ n(x)=(n(x))^{3}\left[(n(x))^{3(\nu-1)}+(n(x))^{3}\right] .
$$

Apply 4.10 together with a suitable induction hypothesis.

Lemmas 4.11-4.19 show that $J(1)$ satisfies conditions $2.01-2.02$. Now we turn to condition 2.03 .

4.20. LemMA. The cosets of $J(1)$ in $A$ are represented by $1, x, x^{3}, 1+x$, $1+x^{3}, x+x^{3}, 1+x+x^{3}$.

4.21. LEMMA. For $\alpha=1,2,3$, if $n(x)$ is any polynomial and if $t \in J(1)$, then $(n+t)^{\alpha}-n^{\alpha}-t^{\alpha}$ is in $J(1)$. 
Proof. For $\alpha=1,2$ this is obvious. For $\alpha=3$, note that $\bmod 2$, $(n+t)^{3} \equiv n^{3}+n^{2} t+n t^{2}+t^{3}$, so that $(n+t)^{3}-n^{3}-t^{3} \equiv n^{2} t+n t^{2}$. Since $\left(n_{1}+n_{2}\right)^{2} \equiv n_{1}^{2}+n_{2}^{2} \bmod 2$, and since $\left(t_{1}+t_{2}\right)^{2}=t_{1}^{2}+t_{2}^{2} \bmod 2$, the form $n^{2} t+n t^{2}$ is additive:

$$
\begin{aligned}
& \left(n_{1}+n_{2}\right)^{2}\left(t_{1}+t_{2}\right)+\left(n_{1}+n_{2}\right)\left(t_{1}+t_{2}\right)^{2} \\
& \equiv n_{1}^{2} t_{1}+n_{1} t_{1}^{2}+n_{2}^{2} t_{2}+n_{2} t_{2}^{2}+n_{1}^{2} t_{2}+n_{2}^{2} t_{1}+n_{1}+n_{1} t_{2}^{2}+n_{2} t_{1}^{2} \\
& \quad \equiv\left(n_{1}^{2} t_{1}+n_{1} t_{1}^{2}\right)+\left(n_{2}^{2} t_{2}+n_{2} t_{2}^{2}\right)+\left(n_{1}^{2} t_{2}+n_{1} t_{2}^{2}\right)+\left(n_{2}^{2} t_{1}+n_{2} t_{1}^{2}\right) .
\end{aligned}
$$

Thus the lemma has to be checked only for the atoms.

4.22. LemMa. For $\alpha \geq 4$, if $n(x)$ is any polynomial and if $t \in J(1)$, then $(n+t)^{\alpha}-n^{\alpha}-t^{\alpha} \in J(1)$.

Proof. The inductive argument proceeds in steps of 3. Modulo 2, note that $(n+t)^{3} \equiv n^{3}+n^{2} t+n t^{2}+t^{3}$. Thus, $\bmod 2$,

$$
\begin{array}{r}
(n+t)^{\alpha}-n^{\alpha}-t^{\alpha} \equiv\left(n^{3}+n^{2} t+n t^{2}+t^{3}\right)\left(n^{\alpha-3}+t^{\alpha-3}+s\right), \\
s \in J(1) .
\end{array}
$$

This uses the inductive hypothesis that $(n+t)^{\alpha-3}-n^{\alpha-3}-t^{\alpha-3}$ is a polynomial $s$ in $J(1)$. There are three cases: $\alpha=0,1,-1 \bmod 3$.

If $\alpha \equiv 0 \bmod 3$, then also $\alpha-3 \equiv 0 \bmod 3$. Complete the proof by referring to $4.10,4.21$.

If $\alpha \equiv 1 \bmod 3$, we assume wolg that the constant term in $t$ is 0 . The terms in the expansion of (4.23) that are not taken care of by 4.10 are

$$
\left(n^{2} t+n t^{2}\right) s+t^{3} s+\left(n^{2} t+n t^{2}\right) t^{\alpha-3}+n^{\alpha-4}\left(n^{3} t+n^{2} t^{2}+n t^{3}\right) .
$$

Of these four terms, the first three are in $J(1)$ by 4.21 , 4.08. It remains to prove that $n^{3} t+n^{2} t^{2}+n t^{3}$ is in $J(1)$. As to $n^{3} t$, see 4.10. Write $n^{2} t^{2}+n t^{3}$ $=\left(n^{2} t+n t^{2}\right) t$ to complete the proof.

If $\alpha \equiv-1 \bmod 3$, again assume wolg that the constant term in $t$ is 0 . The terms in the expansion of (4.23) that require argument are $n^{\alpha-5}\left(n^{4} t+n^{3} t^{2}+n^{2} t^{3}\right)$. It has to be proved that $n^{4} t+n^{2} t^{3}$ is in $J(1)$. By $4.21, n^{4} t \equiv n^{2} t^{2} \bmod J(1)$, and both $n^{2} t^{2}, n^{2} t^{3}$ have zero constant term. So it has to be proved that $n^{2}\left(t^{2}+t^{3}\right) \in J(1)$. This last assertion follows from Lemma 4.24, with the application cited afterwards.

4.24. LeMMA. If $t(x)$ is in $J(1)$ [and if $t(0)=0$ ] then $p(x)=t+t^{2}$ is a polynomial in $x$ with $p(0)=0$ and such that the number of terms with exponent $\equiv 0 \bmod 3$ is even; the number of terms with exponent $\equiv 1 \bmod 3$ is even; the number of terms with exponent $\equiv-1 \bmod 3$ is even. 
Proof. $t_{1}+t_{2}+\left(t_{1}+t_{2}\right)^{2} \equiv\left(t_{1}+t_{1}^{2}\right)+\left(t_{2}+t_{2}^{2}\right) \bmod 2$. Also the assertion of the lemma is valid for each atom (each generator) in $J(1)$.

Application of the lemma. If $p(x)$ has the properties stated, then so also does $m(x) p(x)$, where $m(x)$ is any polynomial, provided $p(0)=0$. Set $m(x)=n^{2} t$.

4.25. THEOREM. if $C$ is a principal ideal ring such that for every $c \in C$, $c+c^{2}$ is a multiple of 2, then the only ideals in the (near ring or) composition algebra $\left(C[x],+,{ }^{\circ}\right)$ are $c C[x], c T(1), c V(1), c J(1)$, with $J(1)$, $V(1), T(1)$ defined as in $2.18,2.21$. The set of inclusion relations is the obvious set, together with those in the diagram below.

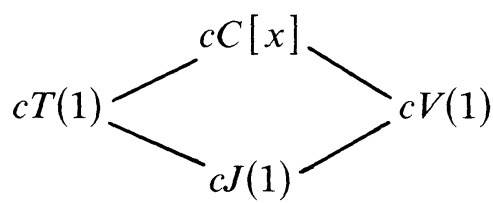

The near-ring (composition algebra) ideals are all principal in this case.

4.26. Corollary. Theorem 4.25 holds if $C$ is the ring of algebraic integers in $Q(\sqrt{-\Delta})$, where $-\Delta$ is any one of $-3,-7,-11,-19,-43$, $-67,-163$.

4.27. ReMARK. If the hypotheses of 4.08 do not hold, then the smallest ideal $J(c)$ containing $c$ contains also

$$
\begin{gathered}
\left\langle c, 2 c x^{\nu},\left(c^{3}+c^{4}\right) x^{\nu},\left(c^{2}+c^{3}\right) x^{2 \nu}, c^{3}\left(x^{3 v+1}+x\right),\right. \\
c^{3}\left(x^{3 \nu-1}+x\right), c^{3}\left(x^{3 \nu}+x^{3}\right), c^{2}\left(x^{6 \nu+3}+x^{3}\right), \\
c^{2}\left(x^{6 \nu+5}+x^{5}\right), c^{2}\left(x^{6 \nu+7}+x^{7}\right), \\
\left(c^{2}+c^{3}\right)\left(x^{2 v+3}+x^{3}\right)|\nu=1,2, \ldots\rangle
\end{gathered}
$$

However, the module with these generators need not be an ideal. (The assertion in 4.27 has a lengthy proof.)

4.28. Problem. Characterize the ideal $J(c)$ in a simple manner.

4.29. Problem. If $C$ is the ring of Gaussian integers, is $J(1)$ the module (over $C$ )

$$
\left\langle 1,2 x^{\nu},(1+i) x^{\nu}, x^{3 \nu+1}+x, x^{3 \nu-1}+x, x^{3 \nu}+x^{3} \mid \nu=1,2, \ldots\right\rangle ?
$$

What are the other ideals in $(C[x],+, \circ)$ ? 
5. Properties of the binomial coefficients. The first two properties are easy.

5.01. LEMMA. $C_{k}^{2 k}$ is even.

5.02. LEMMA. If two of the lower suffixes of a multinomial coefficient are equal and positive, the coefficient is even.

More generally if there are $r$ pairs of positive and equal suffixes, $C_{i, j, k, l}^{n} \ldots$ is divisible by $2^{r} .\left[C_{1,1,1,1}^{4}, C_{2,2,2,2}^{8}, C_{1,1,5,5}^{12}=2^{4} 3^{3} \cdot 7 \cdot 11\right.$ are all divisible by 4.]

The next lemmas all follow from 2.03. A direct proof of 5.03 is immediate; without using 2.03, the others seem less obvious.

5.03. LemMA. Among the $n+1$ binomial coefficients $C_{k}^{n}$, an even number are odd.

5.04. Lemma. Among those binomial coefficients $C_{3 \nu}^{n}$ with $\nu$ an integer and with $0<3 \nu<n$, an even number are odd.

(5.04 is immediate if $n$ is a multiple of 3; 5.04 is true without this restriction.) R. J. Evans showed me a direct proof of 5.04.

5.05. LEMMA. Let $S$ be the collection of those multinomial coefficients $C_{l j k l m}^{n}$ that are odd, and in which $0<i<n$, subject to the further restriction $j+m \equiv k \bmod 3$. Then $S$ has odd cardinality.

5.06. LEMMA. The cardinality of the set

$$
\begin{array}{r}
\left\{C_{i_{0}, i_{1}, \ldots, l_{r}}^{n} \mid 0<i_{0}<n, i_{1}+2 i_{2}+\cdots+r i_{r} \equiv 0 \bmod 3,\right. \\
\left.C_{i_{0}, l_{1}, \ldots, i_{r}}^{n} \text { is odd }\right\}
\end{array}
$$

is even.

5.07. THEOREM. The assertion of the preceding number remains true if the congruence $\sum j \cdot i_{j} \equiv 0 \bmod 3$ is replaced by the congruence $\sum a(j) i_{j} \equiv 0$ $\bmod 3$, where $a(j)$ are arbitrary integers.

This conclusion is obtained by noting that the polynomial $\left(1+\sum_{j>0} x^{a(j)}\right)^{n}-1^{n}-\left(\sum x^{a(j)}\right)^{n}$ must lie in $J(1)$.

Acknowledgment. Professor Denis Floyd made valuable critical suggestions. 


\section{REFERENCES}

[1] J. L. Brenner, Maximal ideals in the near ring of polynomials module 2, Pacific J. Math., 52 (1974), 595-600.

[2] E. G. Straus, Remark on the preceding paper, ideals in near rings of polynomials over a field, Pacific J. Math., 52 (1974), 601-603.

Received July 19, 1984

10 PHILLIPS RD.

Palo Alto, CA 94303 
